\title{
O Impacto dos gestos de Agentes Pedagógicos Animados na compreensão de dicas pelos aprendizes
}

\author{
Igor M. P. Martins ${ }^{1}$, Felipe de Morais, Bruno Luis Schaab, Patricia Jaques \\ ${ }^{1}$ Universidade do Vale do Rio dos Sinos (UNISINOS) \\ Caixa Postal 275 - 93.022-000 - São Leopoldo - RS - Brasil \\ igormpmartins@gmail.com, pjaques@unisinos.br \\ \{felipedemoraisfm,bruno_schaab\}@hotmail.com
}

\begin{abstract}
Intelligent Tutoring Systems (ITS) are powerful computing tools for supporting learning, since they offer customized assistance. However, in most ITSs, the text hints presented by the tutor when student is stuck are not very clear, because they have little connection with the system's visual elements. In this paper, we evaluate if the use of gestures by an APA for explaining hints about equation solving improves the student's understanding of the related abstract concepts. The results of a qualitative evaluation with 4 students demonstrated that students understand more correctly the hints and preferred to use the tutor version with the agent that employs gestures.
\end{abstract}

\begin{abstract}
Resumo. Sistemas Tutores Inteligentes (STIs) são poderosas ferramentas de apoio à aprendizagem, pois oferecem ensino personalizado. Entretanto, na maioria dos STIs, as dicas textuais não são muito claras para os aprendizes, pois estão pouco conectadas com os elementos visuais. Nesse artigo, foi verificado se o uso de gestos de um Agente Pedagógico Animado (APA) para explicar dicas relacionadas à resolução de equações em um STI melhora a compreensão dos estudantes dos conceitos abstratos envolvidos. Os resultados de uma avaliação qualitativa com 4 alunos mostraram que os alunos compreenderam melhor as dicas e preferiram usar a versão do tutor em que o agente emprega gestos.
\end{abstract}

\section{Introdução}

Uma das principais características dos Sistemas Tutores Inteligentes (STIs) é fornecer assistência personalizada aos aprendizes, de acordo com as informações inferidas sobre seu conhecimento e perfil e guardadas no modelo de aluno. Essa assistência se dá basicamente pela correção dos passos de um exercício e também pelo fornecimento de dicas quando o aluno não souber como prosseguir na resolução.

Existem questões de design muito importantes na implementação desse mecanismo de dicas. Deve-se determinar se as dicas serão exibidas quando solicitadas ou quando o STI detectar adivinhações; estabelecer em que tipo de passo deverá aparecer e como as dicas poderão maximizar a experiência e permitir ao aluno resolver o problema [Vanlehn 2006]. Dessa forma, para desenvolver um mecanismo de feedback, não são necessários apenas conhecimentos técnicos sobre tratamento de mensagens. A forma como as informações são apresentadas, o conteúdo a ser passado, e o momento em que cada mensagem é exibida devem ser cuidadosamente estudados. 
No entanto, pesquisas têm mostrado que as dicas em STIs não tem sido completamente efetivas, permitindo estratégias dos alunos que são associadas com baixos desempenhos educacionais. Os alunos continuamente apresentam os comportamentos de help refuse (não aceitar ajuda do sistema) e help abuse (usar de forma abusiva as ajudas disponibilizadas para obter a resposta sem tentar compreender a dica), deixando de aproveitar os benefícios do sistema, e não havendo assim evolução na aprendizagem [Baker et al. 2006].

Estudos apontam que professores humanos empregam gestos para ilustrar e salientar aspectos mais importantes de uma explicação e que isso tem um impacto positivo importante na compreensão pelos alunos [Cook et al. 2013, Goldin-Meadow 1999]. Dessa forma, neste trabalho, supõe-se que, em um STI, os gestos de um agente pedagógico animado (APA) podem ser empregados para ilustrar as dicas e dessa forma melhorar a sua compreensão. APAs são personagens animados em software, que oferecem uma forma de interação similar a um professor real, através de sua comunicação por gestos e expressões faciais. Diferente de simples personagens de desenhos, APAs tem seus próprios objetivos de ensino e fazem decisões sobre quais ações tomar para alcançar esses objetivos.

Neste trabalho busca-se melhorar a compreensão dos alunos de conceitos abstratos, tais como equivalência em equações e operações algébricas, através do uso de gestos em um agente pedagógico animado ao exibir dicas. O APA foi integrado a um STI de álgebra, chamado PAT2Math [Jaques et al. 2013, Seffrin et al. 2012], que assiste o aluno na resolução passo-a-passo de equações, fornecendo feedback mínimo para cada passo e mostrando dicas quando requisitadas pelo aluno ou quando o aluno resolver um passo incorretamente. A proposta do APA é indicar os erros cometidos pelos alunos durante a solução do problema, apontando quais partes da equação possuem erros ou salientando as partes mais importantes durante a explicação. Os gestos do agente foram elaborados de acordo com uma pesquisa específica sobre o uso de gestos por professores humanos no ensino de álgebra. Além disso, foram também analisados vídeos de professores humanos explicando como resolver equações.

Os gestos de APAs tem sido empregados em ambientes educacionais principalmente com o objetivo de reduzir a carga cognitiva do aluno [Dunsworth and Atkinson 2005], chamando a atenção do aluno para materiais relevantes [Atkinson 2002, Johnson et al. 2000, Lester et al. 1997b]. No caso do trabalho proposto, os gestos são empregados em dicas fornecidas pelo agente para elucidar conceitos abstratos, tais como igualdade em equações algébricas. Dessa forma, esse trabalho traz uma importante contribuição no sentido de prover uma alternativa para melhorar a compreensão de dicas dos estudantes em STIs, impactando assim positivamente na aprendizagem dos estudantes. Busca-se igualmente minimizar comportamentos indesejados dos aprendizes, tais como gaming the system, help refuse, help abuse, que mostram ter um efeito negativo na aprendizagem [Baker et al. 2006].

Foi realizada uma avaliação qualitativa com quatro alunos para verificar o impacto do uso de gestos de um APA na compreensão de dicas em um tutor de álgebra. Os resultados mostraram que os alunos entenderam mais corretamente as dicas providas pelo agente quando elas estavam acopladas com gestos. Além disso, os alunos também preferiram usar a versão do tutor na qual o agente empregava gestos. 


\section{Gestos na Aprendizagem}

Existem diferentes fatores que podem influenciar a compreensão dos alunos, entre eles está a comunicação não verbal transmitida pelos professores. Gestos tem um papel importante na aprendizagem. Crianças aprendem mais significativamente ao observar gestos de professores, em particular enquanto aprendem tarefas matemáticas [Cook et al. 2013].

Em um estudo conduzido por [Valenzeno et al. 2003], crianças que observaram um instrutor que fazia gestos enquanto explicava sobre simetria tiverem melhores resultados do que um grupo que não teve gestos na explicação. Um outro estudo realizado por [Cook et al. 2013] avaliou o impacto dos gestos na retenção do conhecimento no ensino de equivalência em equações realizado com 184 crianças, alunos dos $2^{\circ}, 3^{\circ}$ e $4^{o}$ anos. Foram preparados vídeos, nos quais o instrutor explicava como resolver problemas de equivalência matemática utilizando gestos ou não. Cada sala de aula foi aleatoriamente atribuída para o grupo de controle (vídeos apenas com fala) ou para o grupo experimental (gestos e fala). Foi constatado que não apenas houve ganho de aprendizagem no grupo experimental, mas também que houve ganho após 24 horas, independente do conhecimento inicial do aluno.

Um dos motivos que poderia ter levado a esse ganho é a atenção que se traz para a lição ao utilizar gestos. Foi observado, durante instrução com gestos, que os alunos desviavam menos sua cabeça da direção do vídeo do que alunos que tiveram instruções sem gestos [Valenzeno et al. 2003]. Entretanto, é possível que os ganhos não venham apenas do aumento de foco, porque um aluno que tivesse tido níveis similares de foco durante os testes deveriam ter resultados similares. Então, sugere-se que os gestos tem um efeito único na aprendizagem. Uma possibilidade levantada é que gestos podem clarificar ou transmitir informações que não são imediatamente aparentes na fala [Cook et al. 2013].

Há duas principais formas de cooperação entre gestos e falas de um orador: redundância e complementaridade. Redundância é definida como a duplicação da informação em diversas modalidades. Complementaridade é a distribuição da informação através de modos diferentes [Buisine and Martin 2007]. Para ilustrar os dois tipos de cooperação entre gestos e fala, considere uma aula de história sobre a rota de invasão de Hannibal [Buisine and Martin 2007]. Se o professor diz "Hannibal foi do norte da África até a Itália" e faz dois gestos no mapa (um indicando o norte da África e o outro indicando a Itália), os gestos são considerados redundantes em relação ao conteúdo da fala (eles duplicam a identificação dos países mencionados na fala). Da mesma forma, se o professor diz "Hannibal foi do norte da África para lá" e completa a fala apontando para a Itália, o gesto é considerado complementar. $\mathrm{O}$ ouvinte tem que integrar ambas as modalidades para captar a mensagem completa.

Há experimentos que mostram que a redundância entre fala e gestos possuem melhores resultados em agentes animados, porém não há evidências se o mesmo é aplicável no comportamento de tutores humanos [Buisine and Martin 2007].

\section{Trabalhos Relacionados}

Em ambientes educacionais, os gestos de APAs tem sido empregados principalmente com o objetivo de reduzir a carga cognitiva do aluno. Um exemplo seria seu uso como um indicador visual para reduzir a carga de busca cognitiva do aluno 
[Dunsworth and Atkinson 2005], chamando a atenção do aluno para materiais relevantes [Atkinson 2002]. Da mesma forma, os gestos de APAs tem sido empregados para apontar e destacar elementos em ambientes 2D e 3D para ensino através de simulação. Como exemplo desse último caso, podemos citar os agentes Steve [Johnson et al. 2000] e Cosmo [Lester et al. 1997b].

Em um estudo conduzido por [Buisine and Martin 2007], foi implementado um sistema para explicar o funcionamento de três objetos utilizando agentes animados: um software para edição de vídeos, um controle remoto de um projetor e uma fotocopiadora. As principais dificuldades apresentadas pelos usuários eram as ambiguidades relacionadas às posições, cores e formas das teclas ou itens dos menus dos três objetos citados. Esse software foi construído para conseguir medir os efeitos da cooperação entre gestos e falas nos grupos dos usuários. Para isso, foram implementadas três versões do agente Lea: uma que utilizava cooperação redundante, outra que utilizava cooperação complementar e uma outra versão que não utilizava gestos, porém ainda assim o agente estava presente. Nesses testes realizados por [Buisine and Martin 2007], constatou-se que houve maior aprendizagem ao utilizar a forma de cooperação redundante.

Nos trabalhos relacionados, os gestos de agentes são empregados para destacar objetos físicos presentes no mundo virtual (simulado ou não) e/ou diminuir a carga cognitiva do aluno. No trabalho proposto, os gestos são empregados em dicas fornecidas pelo agente para elucidar conceitos abstratos, tais como operações algébricas. Não se tem conhecimento de outros trabalhos que buscaram avaliar o impacto dos gestos de um APA para explicar dicas. Além disso, mesmo que existam outros tutores voltados ao ensino de álgebra [Koedinger et al. 1997], não temos conhecimento de nenhum trabalho que tenha aplicado os gestos de um APA para melhorar a compreensão dos estudantes de conceitos abstratos das dicas sobre resolução de equações.

\section{Trabalho Desenvolvido}

Como explicado na Seção 1, o trabalho proposto busca melhorar a compreensão das dicas pelos alunos através do uso de gestos de um agente pedagógico animado (APA). $\mathrm{O}$ APA foi integrado ao STI web de álgebra chamado PAT2Math ${ }^{1}$, que assiste o aluno na resolução passo-a-passo de equações algébricas de $1^{\circ}$ e $2^{\circ}$ grau com uma incógnita, voltado para alunos de $6^{\circ}, 7^{\circ}$ e $8^{\circ}$ anos [Jaques et al. 2013]. A interface do módulo para resolução de equações deste tutor pode ser observada na Figura 1(a). A assistência do APA se dá de duas formas: realizando a correção em tempo-real do passo do aluno, provendo feedback mínino (certo ou errado) e fornecendo dicas (mensagens de ajuda). As dicas podem ser exibidas tanto quando o aluno fornecer um passo incorreto ou quando ele solicitar (i. e. porque não sabe o próximo passo).

$\mathrm{Na}$ versão anterior do tutor, as dicas eram exibidas apenas na forma de texto em um balão de texto do agente, assim como numa caixa de texto que fica permanentemente na tela para que o aluno possa se lembrar da dica. Ao exibir uma dica ou erro, não há qualquer destaque na equação do passo atual, apresentada na interface do tutor, dos elementos mencionados nas mensagens de texto. Isso pode ser observado na interação exemplificada na Figura 1(a). O aluno pediu uma dica pois não sabia que resposta fornecer como próximo passo para a equação a ser resolvida. Foi exibida uma mensagem textual ao

\footnotetext{
${ }^{1}$ Disponível em http://pat2math.unisinos.br/
} 


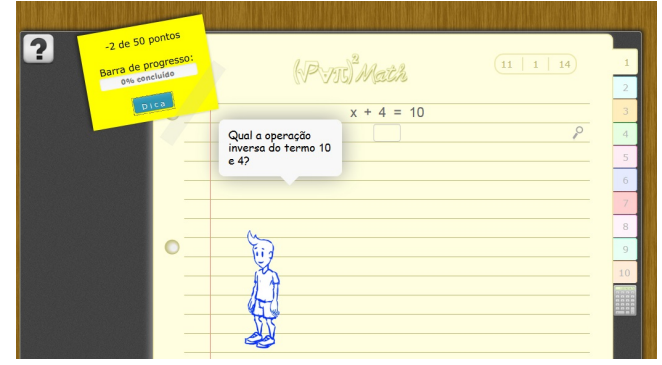

(a) Sem gestos

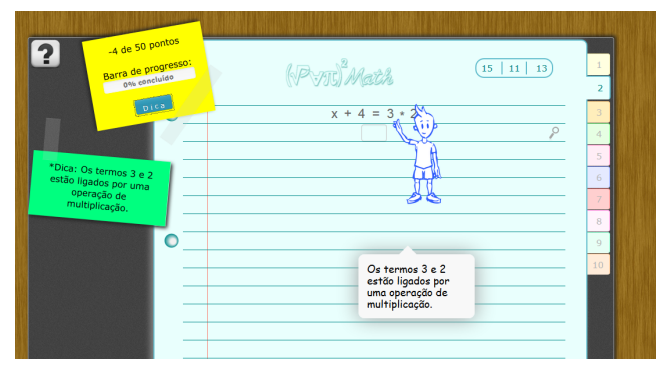

(b) Com gestos

Figura 1. Versão anterior e atual do APA

aluno (mostrada no balão de texto do agente), porém os termos da equação mencionados (os números 10 e 4) não são destacados no quadro, assim como não são realizados destaques na interface do STI.

Na versão anterior do tutor, o agente não possuía inteligência e sua função no tutor consistia basicamente em mostrar as dicas no balão de texto, saudar o aluno, parabenizalo quando tivesse finalizado a resolução de uma equação e mostrar algumas mensagens de encorajamento quando o aluno fizesse sucessivas tentativas incorretas de respostas. Para o trabalho proposto, foi implementada uma nova versão do agente pedagógico. Ao explicar as dicas, além de exibir o conteúdo textual, o novo agente utiliza gestos para indicar, na equação do passo corrente, exibida na interface, os elementos mencionados no texto da ajuda. Como pode ser observado na Figura 1(b), a mesma mensagem de dica exibida na versão anterior (Figura 1(a)) recebe um tratamento diferenciado na interface. Ao efetuar a explicação dessa dica, o agente aponta para os elementos 3 e 2 que estão na tela, empregando os gestos redundantemente com o conteúdo da fala. $\mathrm{O}$ agente tem ações diversas pré-definidas que são exibidas de acordo com o conteúdo da dica. Para certas mensagens, o agente pode apenas apontar para a equação, assim como pode destacar determinados elementos da equação. Há um conjunto de 10 animações pré-definidas, que estão detalhadas na Seção 4.1.

Além da interface apresentada na Figura 1(b), o tutor possui também um sistema especialista baseado em regras de produção que realiza as funções de Step Generator e Step Analyser [Vanlehn 2006]. O Step Generator, dada uma equação corrente, é capaz de determinar próximos possíveis passos (solução). O Step Analyser compara o passo fornecido pelo aluno com as possíveis respostas do Step Generator para verificar se o passo está correto. Como o tutor possui também uma biblioteca de erros, o sistema pode também identificar falsas concepções do estudante. Para a exibição das dicas, a interface realiza uma requisição ao Step Analyser, passando como parâmetro a equação que está sendo resolvida pelo aluno. Após o processamento ser concluído pelo Step Analyser, a interface interpreta os resultados e os exibe.

O módulo de dicas guarda as dicas textualmente, organizada em níveis e por operação algébrica. Assim, para cada operação, há geralmente 5 níveis de dicas que compreendem dicas mais genéricas (por exemplo, chamar atenção do aluno para um termo) a dicas mais específicas (por exemplo, chamar o Step Generator para fornecer a resposta ao aluno). Para que as dicas pudessem ser customizadas para cada passo, os textos de ajuda usam marcas e templates que permitem substituir determinadas marcas por termos 
específicos do passo corrente.

As mensagens de dicas incluem igualmente a identificação do elemento que deve ser destacado gestualmente pelo agente através de tags. Um exemplo do novo formato de mensagem é: "Os termos $<$ eq id $=0>3</ e q>e<e q i d=1>2</$ eq $>$ estão ligados por uma operação de multiplicação". Com isso, o agente consegue determinar que os elementos com $i d 0$ e 1 devem ser destacados na interface, durante a execução de uma das animações pré-definidas. Além disso, para tornar viável a explicação gestual das dicas, foi necessário criar um vínculo entre as mensagens de dicas e um conjunto de animações pré-definidas. Então, ao efetuar a requisição de uma dica, o módulo de dicas devolve entre as informações o código da animação pré-definida. O módulo de dicas utiliza um modelo de aluno que guarda a lista de operações empregadas pelo aluno em cada passo e os respectivos níveis de conhecimento e uma lista de travas por operação, utilizada para evitar o help abuse.

O APA foi desenvolvido utilizando o framework Divalite. Esse framework foi escolhido pois oferece uma integração fácil, rápida e robusta com aplicações web, como o caso do tutor. O Divalite funciona através da manipulação do Document Object Model (DOM) em uma interface HTML5. Isso permite que o agente possa apontar termos específicos da equação (por exemplo, o termo 3 ou 2 na equação da Figura 1(a)), uma vez que cada termo é representado como um objeto DOM e, por isso, sua localização na tela é conhecida. Embora existam alguns personagens disponíveis no DivaLite, optou-se por criar um novo agente devido à identidade visual da aplicação (Figura 1(a)). Isso permite também estender os comportamentos do agente quando necessário mais facilmente, uma vez que, no DivaLite, cada comportamento é representado por uma sequência de figuras. De fato, para o trabalho proposto, foram desenhadas novas animações necessárias para o agente apontar para elementos específicos da equação. Para um efeito mais natural, o APA sempre fica presente na interface do tutor, com movimentos de idle (por exemplo, piscar, cocar a cabeça, etc) aleatórios pré-definidos para dar mais realismo ao agente.

\subsection{Definição dos Gestos do agente}

Antes da definição de quais animações seriam executadas pelo APA, foi efetuada uma análise sobre a comunicação não verbal de professores ensinando matemática. Para haver uma proximidade maior com o trabalho aqui apresentado, foram analisados vídeos de professores ensinando como resolver equações de $1^{\circ}$ grau. Isso também ajudou a isolar os elementos comuns entre os diferentes professores, pois em todos os vídeos eles estavam ensinando o mesmo conteúdo, apesar de haver algumas pequenas diferenças, devido suas particularidades para explicar o conteúdo.

Os vídeos foram selecionados da plataforma youtube . com, empregando como critério de seleção as notas e popularidade, dentro do assunto equações de $1^{o}$ grau. Foram analisados vídeos de 5 professores diferentes, sendo que 2 desses professores eram estrangeiros. Houveram outros 5 vídeos inicialmente selecionados pelas avaliações e popularidade, porém foram descartados por não mostrarem gestos.

Um elemento comum observado em todos os vídeos analisados era destacar no quadro os elementos que estavam sendo mencionados na fala do professor, o que se carateriza uma cooperação redundante entre gestos e fala. Outro elemento comum foi a posição neutra durante a explicação de conceitos. Em particular nessa situação, os profes- 
sores costumavam olhar em direção ao expectador; em alguns casos até mesmo olhando fixamente para o aluno por alguns segundos, para então seguir em sua explicação.

Após a análise dos vídeos, foi feita uma análise entre as mensagens de dicas já existentes no módulo, isolando elementos comuns encontrados entre as dicas. Com isso, foram propostos 10 tipos de animações que o agente deve executar para ilustrar as dicas. Eles seguem a cooperação redundante entre fala e gestos, que mostrou melhores resultados em trabalhos relacionados [Buisine and Martin 2007]. Isso também procede com [Goldin-Meadow 1999], que mostrou que crianças tem uma melhor performance em problemas matemáticos quando os gestos de mão dos professores são correspondentes a sua fala. Graças a análise dos vídeos, foi possível ter um parâmetro para aproximar os gestos do agente com os gestos que os professores utilizam. Através da definição dessas animações, foi possível vincular uma animação para cada uma das dicas já existentes. A seguir, listamos as animações gestuais criadas.

- O APA aponta para cada um dos termos da equação. É utilizada para casos em que se deseja que o aluno note alguma característica ou elemento da equação.

- O APA aponta para elementos específicos da equação. É empregada para destacar uma operação que o aluno deve realizar entre dois termos.

- O APA apenas aponta para a equação como um todo, sem destaques. É usada para indicar para o aluno que uma determinada operação poderá ser realizada, quando é desejável que o aluno por si descubra em que termos ele deve aplicar a operação.

- O APA aponta para determinados termos da equação e no final aponta para um operador. Útil para destacar qual operação deverá ser realizada entre determinados operandos.

- O APA aponta para determinados termos e no final aponta para o próprio balão de dicas. Essa animação é útil para indicar um resultado de uma operação sobre termos, pois o APA aponta os termos e no final destaca o resultado no balão.

- O APA aponta para as frações existentes na equação. Útil para indicar a aplicação de MMC.

- O APA aponta para cada lado da equação. Utilizado para indicar operação inversa ou salientar o conceito de igualdade.

- O APA aponta para todas as operações existentes na equação. Utilizada para destacar as operações que o aluno deve fazer, ou alertar caso o aluno tenha empregado uma operação incorretamente.

- O APA aponta para o resultado incorreto do aluno. Utilizada para destacar que o aluno deve revisar a resposta dada.

- O APA apenas mantém a posição neutra das mãos. Utilizada quando ele revisa algum conceito, sem necessidade de efetuar destaques para a equação.

\section{Avaliação}

Foi realizada uma avaliação qualitativa do trabalho proposto com o objetivo de verificar a compreensão dos alunos das dicas quando empregados os gestos redundantes do APA. Essa etapa da avaliação consistiu na utilização do STI PAT2Math por quatro alunos da sétima série do ensino fundamental de uma escola privada da Grande Porto Alegre, seguida de uma entrevista composta essencialmente por questões abertas. Foi solicitado à professora dos alunos que selecionasse estudantes com desempenho variado em álgebra: 
ótimo (1 aluno), médio (2) e baixo (1). Cada aprendiz interagiu com duas versões do sistema. Na primeira versão, as dicas eram explicadas pelo APA sem ajuda gestual, ou seja, apenas através de texto exibido no balão de mensagem do agente e também destacado em um post-it de dicas na tela. Na segunda versão, além da presença do agente, o mesmo apontava para elementos da equação de acordo com o texto da dica apresentada. Foi mantida a presença do agente em ambas as versões, pois o objetivo era verificar se a preferência pela versão do agente com gestos, e a melhor compreensão das dicas, se deve aos gestos do agente ou apenas a sua presença, chamada de Image Effect [Lester et al. 1997a]. A comunicação não verbal do agente na segunda versão, através de gestos, utilizou a forma de cooperação entre gestos e fala denominada redundante, pois estudos apontam a melhor efetividade dessa abordagem [Buisine and Martin 2007]. As sessões tiveram duração de 2 horas ao total, sendo que em média os estudantes interagiram 20 minutos com cada versão.

Inicialmente, os sujeitos foram informados sobre os objetivos do experimento, o caráter voluntário de sua participação e o completo sigilo mantido sobre quaisquer dados individuais coletados. Sobre o experimento, foi apenas comentado que eles utilizariam duas versões de um ambiente inteligente de aprendizagem para ensinar álgebra e o objetivo era verificar qual era a melhor versão. Os alunos também foram notificados que poderiam abandonar a pesquisa antes e durante seu curso, sem prejuízo para si. Além disso, como a faixa etária dos alunos era de 12 a 14 anos, foi solicitado uma semana antes da sessão que os pais assinassem o Termo de Consentimento Livre e Esclarecido (TCLE). O projeto da avaliação, o TCLE, assim como a carta de consentimento da escola foram submetidos na Plataforma Brasil e aprovados pelo Comitê de Ética da universidade dos autores.

No início da sessão, os alunos tiveram uma pequena explicação sobre o funcionamento do ambiente. Após, eles resolveram em média 4 equações em cada uma das versões do sistema. Em seguida, foi realizada a entrevista. Todas as sessões foram registradas de duas maneiras: (i) foi gravada a interação dos alunos com as versões do sistema através de software de captura de tela, (ii) foi gravado som e imagem do busto e rosto dos alunos através da webcam do notebook usado para o experimento. Dois experimentadores se intercalaram para realizar a entrevista com os alunos. Os experimentadores se mantiveram ao lado dos estudantes durante o período em que eles interagiram com o sistema, porém, não forneciam ajuda de como resolver as equações. Toda vez que o aluno informava que não sabia como proceder, o experimentador sugeria que o aluno solicitasse uma dica ao sistema. Caso o aluno ainda não soubesse como prosseguir após receber as dicas, o experimentador solicitava que o estudante resolvesse a próxima equação. Para a análise aqui apresentada, foi primeiramente realizada a transcrição de todos os vídeos para texto.

\subsection{Resultados}

De uma forma geral, os alunos consideraram que a versão que utilizava o agente com gestos possuía dicas mais úteis e compreensíveis. Isso foi observado tanto nas respostas da entrevista, como também por falas dos alunos durante a interação com a segunda versão (com gestos) do sistema. Houve apenas um único aluno que não preferiu a segunda versão, porém esse mesmo aluno considerou as dicas da segunda versão mais úteis e compreensíveis: “'Sim, ajudou mais. Talvez porque as questões eram mais difíceis". No caso específico desse aluno, ocorreu o help refusal [Vanlehn 2006], pois ele, mesmo apre- 
sentando dificuldades para a resolução do problema, não solicitava dicas, ou as ignorava e continuava tentando adivinhar o resultado. Dessa forma, como o aluno não solicitava dicas, ele não conseguiu observar diferença entre as duas versões do sistema.

Durante a execução dos testes, houve um aluno que espontaneamente viu a diferença entre os agentes e destacou que as dicas ficavam melhores devido aos gestos ("Melhor esse daqui, daí ele vem aqui e bota a mãozinha em cada número"). Ao serem perguntados de forma explícita qual versão do software tinham preferência, os três alunos que preferiram a nova versão apresentaram respostas similares ("Pelo fato do bonequinho ali, por estar apontando", "O bonequinho se movimenta e mostra ali, fica mais claro", "O agente nos mostra aonde tem que resolver, multiplicar, diminuir na conta"). Nas três respostas, os alunos destacaram que a preferência pela segunda versão ocorreu devido à presença de gestos do agente pedagógico.

Com exceção do aluno que apresentou help refusal, todos consideraram a versão com gestos melhor e mais agradável, principalmente pelo fato de considerarem que as dicas ficaram mais claras. Essa opinião pode ser encontrada na resposta de um dos alunos, que ao ser questionado sobre diferenças entre as versões, respondeu "Eu notei que ele se mexe e a dica [fica] mais clara", mesmo que a pergunta fosse restrita apenas a diferença das versões. Também os alunos conseguiram indicar que essa sensação da nova versão ser mais agradável e melhor ocorreu devido ao uso de gestos, por exemplo, "acho até que ficou mais claro, pois ele chega assim e mostra ali o desenvolvimento".

\section{Conclusão e Trabalhos Futuros}

Nesse trabalho, buscou-se uma forma para melhorar a compreensão dos alunos de conceitos abstratos da matemática, tais como operações algébricas e equivalência em equações. Em busca desse objetivo, foi proposto e implementado o uso de gestos em um APA ao exibir dicas em um STI algébrico. Através do uso de gestos, o APA passou a apontar para as partes da equação relacionadas com a sua explicação ou que possuem erros.

Tanto o uso de gestos no ensino da matemática [Goldin-Meadow 1999], quanto o uso de APAs com gestos com cooperação redundantes [Buisine and Martin 2007] levam a ganhos de aprendizagem. Porém, não foram encontrados trabalhos que mostrassem o impacto de gestos de APAs na compreensão de conceitos abstratos em dicas por estudantes. Esse é o principal diferencial do trabalho proposto.

Foi realizada uma avaliação qualitativa com observação e entrevista a 4 alunos com desempenho variado em álgebra. Os alunos interagiram em média 20 minutos com duas versões diferentes do sistema: (i) versão com dicas apresentadas em balão de texto, (ii) versão com dicas apresentadas pelo agente com texto e gestos redundantes. O objetivo da avaliação era verificar a compreensão dos alunos das dicas quando o agente empregava gestos. Nos resultados coletados, todos os alunos afirmaram que eles compreenderam melhor as dicas na versão na qual o agente utiliza gestos.

Como trabalho futuro, pretende-se realizar uma avaliação experimental para medir os ganhos de aprendizagem obtidos pelos estudantes através do uso de gestos de um APA para explicar dicas. Através de uma avaliação experimental, com grupos de controle e experimental, será possível verificar uma relação causal entre o uso dos gestos pelo agente para explicar as dicas e a compreensão dos alunos das dicas, assim como no seu desempenho. 


\section{Agradecimentos.}

O presente trabalho foi realizado com o apoio do CNPq.

\section{Referências}

Atkinson, R. (2002). Optimizing learning from examples using animated pedagogical agents. Journal of Educational Psychology, 94, pages 416-427.

Baker, R. S. J. D., Corbett, A. T., Koedinger, K. R., Evenson, S., Roll, I., Wagner, A. Z., Naim, M., Raspat, J., Baker, D. J., and Beck, J. E. (2006). Adapting to when students game an intelligent tutoring system. In Proceedings of the 8th International Conference on Intelligent Tutoring Systems, pages 392-401. Springer-Verlag.

Buisine, S. and Martin, J.-C. (2007). The effects of speech-gesture cooperation in animated agents' behavior in multimedia presentations. Interacting with Computers, pages 484-493.

Cook, S. W., Duffy, R. G., and Fenn, K. M. (2013). Consolidation and Transfer of Learning After Observing Hand Gesture. Child development, 24(6):1863-1871.

Dunsworth, Q. and Atkinson, R. K. (2005). Fostering multimedia learning of science: Exploring the role of an animated agent's image. Computers \& Education, 49(3):677690.

Goldin-Meadow, S. (1999). The role of gesture in communication and thinking. Trends in cognitive sciences, 3(11):419-429.

Jaques, P., Seffrin, H., Rubi, G., Morais, F., Ghillardi, C., Isotani, S., and Bittencourt, I. (2013). Rule-based expert systems to support step-by-step guidance in algebraic problem solving: the case of the tutor pat2math. Expert Systems with Applications, $v$. $40, n$. 14 , pages $5456-5465$.

Johnson, W. L., Rickel, J., and Lester, J. (2000). Animated Pedagogical Agents: Face-toFace Interaction in Interactive Learning Environments. IJAIED, 11:47-78.

Koedinger, K. R., Anderson, J. R., Hadley, W. H., and Mark, M. A. (1997). Intelligent Tutoring Goes To School in the Big City. IJAIED, 8:30-43.

Lester, J. C., Converse, S. A., Kahler, S. E., Barlow, S. T., Stone, B. A., and Bhoga, R. S. (1997a). The persona effect: Affective impact of animated pedagogical agents. Proceedings of Computer-Human Interfaces.

Lester, J. C., Voerman, J. L., Towns, S. G., and Callaway, C. B. (1997b). Cosmo: A lifelike animated pedagogical agent with deictic believability. Proceedings of Workshop on Animated Pedagogical Agents, pages 61-69.

Seffrin, H., Rubi, G., de Morais, F., Ghilardi, C., Jaques, P. A., Isotani, S., and Bittencourt, I. (2012). Dicas inteligentes no Sistema Tutor Inteligente PAT2Math. In Simpósio Brasileiro de Informática na Educação, Rio de Janeiro. SBC.

Valenzeno, L., Alibali, M. W., and Klatzky, R. (2003). Teachers' gestures facilitate students' learning: A lesson in symmetry. Contemporary Educational Psychology, 28(2):187-204.

Vanlehn, K. (2006). The Behavior of Tutoring Systems. IJAIED, 16(3):227-265. 